\title{
Composite Events in Chimera
}

\author{
Rosa $\mathrm{Meo}^{1} \quad$ Giuseppe Psaila ${ }^{1} \quad$ Stefano Ceri ${ }^{2}$ \\ 1 Politecnico di Torino, Dipartimento di Automatica e Informatica \\ Corso Duca degli Abruzzi, 24 - I-10129 Torino, Italy \\ 2 Politecnico di Milano, Dipartimento di Elettronica e Informazione \\ Piazza L. Da Vinci, 32 - I-20133 Milano, Italy \\ rosimeo@plito.it, psaila/ceri@elet.polimi.it
}

\begin{abstract}
In this paper, we extend event types supported by Chimera, an active object-oriented database system. Chimera rules currently support disjunctive expressions of set-oriented, elementary event types; our proposal introduces instance-oriented event types, arbitrary boolean expressions (including negation), and precedence operators. Thus, we introduce a new event calculus, whose distinguishing feature is to support a minimal set of orthogonal operators which can be arbitrarily composed. We use event calculus to determine when rules are triggered; this is a change of each rule's internal status which makes it suitable for being considered by the rule selection mechanism.

The proposed extensions do not affect the way in which rules are processed after their triggering; therefore, this proposal is continuously evolving the syntax and semantics of Chimera in the dimension of event composition, without compromising its other semantic features. For this reason, we believe that the proposed approach can be similarly applied for extending the event language of other active database systems currently supporting simple events or their disjunction.
\end{abstract}

\section{Introduction}

Active database systems provide tight integration of Event-Condition-Action (ECA) rules within a database system. Such a tight integration is normally achieved by reusing database system components for implementing conditions (database predicates or queries) and actions (database manipulations, often embedded within a procedural component). In general, when a rule is selected for execution (or triggered), then its condition is evaluated (or considered), and if the condition is satisfied, then the action is immediately executed ${ }^{3}$. Thus, the condition-action (CA) components of an active database have a simple and uniform behavior, which is common to most active databases.

Instead, event type specification, evaluation, and coupling to conditions and actions have to be designed and implemented specifically for each active database system. Thus, it is not surprising that the notions of elementary event type, of event type composition, and of binding between event occurrences and the CA

\footnotetext{
${ }^{3}$ An exception is HiPAC [9] which supports several coupling modes between conditions and actions.
} 
components are quite different in each active database, and such differences are responsible for most of the diversity of active databases.

Most active databases recognize just data manipulation operations (such as insert, delete, and update) as event types. The proposed SQL3 standard, currently under development by ANSI and ISO, associates to each rule just one event type; this can be considered as the simple extreme of a spectrum of solutions [17]. Most relational database products supporting active rules (called triggers) associate each of them to a disjunction of event types whose instances are relative to the same table [23]; this solution is also used by Starburst [24], Postgres [21], and Chimera, an active object-oriented database prototype developed at Politecnico di Milano in the context of the IDEA Esprit Project $[4,5]$. More complex event calculus are supported by active database prototypes (see Section 1.1). In these approaches, rules are associated to event expressions which normally include generic boolean expressions, precedence operations, and explicit time references.

In all active rule systems, event instances cause rules to change an internal state; the corresponding state transition is called triggering of the rule. Once a rule is triggered, active rule systems react in several ways. When multiple rules are triggered at the same time, a rule selection mechanism determines which of them should be considered first; this mechanism may be influenced by priorities which are statically associated to rules. In addition, the rule selection may occurr immediately after the triggering operation or be deferred to some later point in transaction execution (such as the commit time). With immediate execution, it is possible to further identify the cases of rules executing before, after, or instead of the operation generating the triggering event occurrence. Finally, the triggering and execution of rules can be repeated for each tuple or object affected by an operation (row-level granularity in [17]) or instead relate to the overall set of tuples or objects manipulated by means of the same operation (statement-level granularity in [17]).

Due to all these alternatives, active rule systems present themselves with a variety of possible behaviors (a thorough comparative analysis of semantics supported by active rule systems is presented in [10]). In order to control the introduction of complex events in Chimera, and therefore the increase of semantic complexity due to this extension, we have strictly followed some design principles:

- We have defined the event calculus by means of a minimal set of orthogonal operators.

- The semantics of the event calculus is given simply by defining the conditions upon which rules having as event type a complex event calculus expression become triggered; detriggering occurs when a rule is selected for consideration and execution. No other state transitions characterize the internal state of each rule.

- The event calculus extension does not affect the way in which rules are processed after their triggering; therefore, this proposal continuously evolves the syntax and semantics of Chimera in the dimension of event type composition, without compromising its other semantic features. 
We believe that these design principles are general and should drive the design of event calculus for active databases; therefore, we also believe that the proposed approach extends naturally to active database systems currently supporting simple event types or their disjunction.

The paper is organized as follows: Section 2 reports the current fundamental Chimera features; Section 3 introduces the proposed extension, while Section 4 formally gives its semantics; Section 5 deals with implementation issues; finally, Section 6 draws the conclusions.

\subsection{Related work}

There exist several Active Database Systems that have been provided with a language for event type composition; these languages are presented in [13], [7], [11], [19], [22]. The way all these proposals deal with composite event types is quite different depending on the particular systems; in fact, though they have similar sets of operators, different semantics have been proposed. In the rest of the section, we briefly discuss the most important proposals.

Ode [13] has a rich event language based on a small set of primitive operators. These operators deal with event occurrences in a set-oriented way, using set operations like intersection and complement: they produce subsets of the primitive event occurrence history, considered as an ordered set based on the event occurrence time-stamps. For example, event conjunction is the set of event occurrences that satisfy both component event types (and it produces a not null result provided that the two event occurrence sets corresponding to the two operands have at least one common element); event negation is the complement with respect to the whole history; relative of an event type $A$ with respect to type $B$ is the set of occurrences of type $B$ subsequent to the first occurrence of type $A$. Other operators, like event disjunction, temporal precedence (prior), strict sequence (sequence), etc., are derived from the primitive operators. The user is allowed to specify conditions on event properties directly in the composition expressions, i.e. in the event part of the rule. Since the expressive power is that of regular expressions, composite events are checked by means of a finite state automata.

HiPAC [8] makes available data manipulations events, clock events and external events. Clock events can be specified as absolute, relative and periodic. Composite event types are defined with the use of the following operands: disjunction, sequence (temporal precedence of event signals) and closure (event signals occurred one or more times).

Snoop [7] interprets an event $E$ as a boolean function defined on the time domain that is true at time $t$ if an event occurrence of that event type occurs at time $t$. Event conjunction and disjunction are obtained by the boolean algebra applied on their operands. Negation of an event $E$ is defined as the absence of an occurrence of $E$ in a closed interval determined by two events $E_{1}$ and $E_{2}$. While the aperiodic operator determines the presence of all the occurrences of an event $E$ between two subsequent occorrences of $E_{1}$ and $E_{2}$, the periodic operator is equivalent to a periodic event generator: given a time period $t_{p}$, it is true at 
instants separated each other by $t_{p}$, starting from an occurrence of an event $E_{1}$ and stopping at the subsequent occurrence of an event $E_{2}$. The cumulative versions of these two last operators are defined as "accumulating" respectively occurrences of $E$ and time instants. Depending on the application, it is possible to define different contexts in order to let a rule be triggered in correspondance of either all the possible combinations of primitive event occurrences matching the event expression or only some of them.

Samos [11] has a rather rich language as well, which provides the usual event disjunction, conjunction and sequence (ordered conjunction). A Times operator returns the point in time when the $n$-th occurrence of a specified event is observed in a given time interval. The negation is defined as the absence of any occurrence of an event type from a given time interval, and occurs at its end point. A star (*) operator returns the first occurrence of a given event type, regardless of the number of occurrences. Samos allows information passing from the event to the condition part of the rule by means of parameters like the identifier of the transaction in which a given event occurred $\left(c_{-} t i d\right)$, or the point in time of the event occurrence (occ_point). Composite event parameters are derived: disjunction and star $(*)$ receive the parameters of the component event occurrences, conjunction and Times their union. A keyword same specifies that the component events of a composition must have the same parameters.

The Reflex system [19] is an active database system designed for knowledge management applications. Its event algebra provides operators similar to those of Samos. These operators can be classified as logical (and, or, xor and not) or temporal (precedes, succeeds, at, between, within time-spec, every time-spec, etc..)

IF $\mathrm{O}_{2}[22]$ is a conceptual model designed to capture both the structural and behavioural aspects of the modeled reality. The behavioural nodel is based on the notion of event, that represents either a fact of the modelled system which occurs in a spontaneous manner (in the case of external or temporal events) or is generated by the application. The event constructors are composition (conjunction), sequence (temporal precedence), grouping (collection of events of the same type) and union (disjunction). When a $I F \mathrm{O}_{2}$ schema is defined, it is possible to translate it into a set of $E C A$ rules by means of an ad hoc algorithm.

\section{Introduction to Chimera}

Chimera is a novel active, object-oriented and deductive database system; the main goal of its design was the definition of a clear semantics, especially for those aspects concerning active rules, such as rule execution, coupling modes, triggering.

Chimera active rules (also called triggers) follow the ECA (Event-ConditionAction) paradigm. Each trigger is defined on a set of triggering events, and it becomes active if any of its triggering events occurs. The Chimera event language was designed to consider only internal events, i.e. events generated by updates or queries on the database, like create, modify, delete, generalize, specialize, select, 
etc.. In particular, a rule is defined either as targeted or untargeted: if targeted to a class, only events regarding that class are considered for triggering, otherwise events regarding any class in the database can appear in the event part of the rule.

The condition part is a logical formula that may perform a query on the database; its evaluation is called consideration. Depending on the success of this evaluation, the action part is executed coupled with the condition part.

Chimera does not permit binding transfer from the event section to the condition section because of the set-oriented approach; nevertheless, it is important for conditions to obtain objects affected by occurred events. Thus, a condition may include event formulas, particular formulas that query the event base and create bindings to the objects affected by a specified set of event types. Two predicates are available to write event formulas: the occurred predicate and the holds predicate. The former one extracts all the objects affected by the specified event types; the latter considers event composition.

A rule is triggered as soon as one of the triggering events arises, and it is no longer taken into account for triggering, until it has been considered. The triggering mechanism checks for new triggered rules immediately after a non interruptable execution block (either a user instruction sequence, called transaction line, or a rule action).

Based on the Event-Condition (EC) coupling mode chosen by the user, the rule behaves differently: if the rule is defined as immediate, the consideration is performed as soon as possible after the termination of the non interruptable block that generated the triggering event occurrence; if the rule is deferred, it is suspended until the commit command is given.

After the triggering mechanism has checked for new triggered rules, it chooses a rule to be considered and possibly executed, if there is any triggered rule; the choice is made based on a partial order derived from rule priorities provided by the user. Notice that after the consideration and possibly the execution of the rule, it is detriggered and it can be triggered again only by new event occurrences, because events occurred before the consideration loose the capability of triggering the rule.

The user can influence the behaviour of the rule specifying the Event Consumption mode as either consuming or preserving: in the former case, only event occurrences more recent than the last consideration of the trigger are accessible to event formulas; in the latter, all the events occurred since the beginning of the transaction are available.

Example The following rule reacts to the creation of stock items, to check whether the quantity exceeds the maximum quantity admitted for that item.

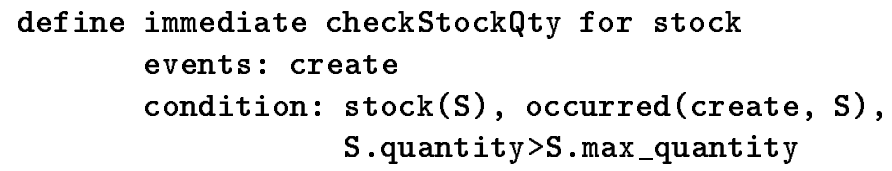


action: modify(stock.quantity, S, S.max_quantity)

end;

The rule, called checkStockQty is defined with immediate EC coupling mode and is targeted to the stock class. The event part indicates that the rule is triggered when a create event on class stock occurs. The condition is structured as follows: a variable $\mathbf{S}$ is defined on class stock; the occurred predicate binds the objects affected by the creation to that variable and finally the constraint is checked. If there is some object that violates the constraint, then the action changes its quantity setting it to the maximum quantity for that object. Note that the rule is executed in a set-oriented way, so all the objects created and not checked yet by the rule are processed together in a single rule execution.

\section{Extending Chimera with Composite Events}

Our extension of Chimera with composite event types moves from the currently available features in order to preserve the characterizing aspects of this system. In particular, the introduction of an event calculus language should change neither the triggering/detriggering semantics, nor the processing of triggered rules, in particular with respect to $E C$ compling modes and event consumption.

A composite event is an event expression obtained from primitive event types by means of a set of operators, such as conjunction, disjunction, negation and temporal precedence. These operators are divided in set-oriented and instanceoriented operators: in the former case, we consider the occurrence of a combination of event types independently of the affected objects; in the latter, the specified combination must occur on the same object. They are reported in Figure 1 , listed in decreasing priority order: set-oriented operators have lower priority than instance-oriented ones, and conjunction and precedence operators have the same priority. A complete introduction to the event calculus language follows in next Sections 3.1 and 3.2. While designing the language, we moved on three othogonal dimensions, as depicted in Figure 2: due to the boolean dimension, we introduced operators such as conjunction, disjunction and negation; due to the granularity dimension, these operators are divided in instance-oriented and setoriented; due to the temporal dimension we introduced two precedence operators, one instance-oriented and the other set-oriented.

In the following two Sections, we introduce the set of operators. For each event expression built by means of each operator, we indicate whether the event has occurred (we say that the event is active) and we indicate the most recent time when the event has occurred (called its activation time-stamp). We make use of some sample event expressions based on classes stock, describing stock products, and show, indicating products on shelves in a sale-room.

\subsection{Set-Oriented Operators}

A primitive event occurs when an occurrence of that event type arises, independently of the object affected by it. For instance, let us imagine that two 
occurrences of the event create (stock) arise at time $t_{1}$ and $t_{2}$. At time $t<t_{1}$ the event is not active; at time $t_{1} \leq t<t_{2}$ the event is active and its activation time-stamp is $t_{1}$; finally, at time $t_{2} \leq t$ the event is active and its activation time-stamp is $t_{2}$.

The first version of Chimera already provided the disjunction among primitive events, that was described by a list of primitive event types separated by commas. We keep the same notation, extending its application to generic event expressions. Intuitively the disjunction $\mathcal{E}_{1}, \mathcal{E}_{2}$ of two event expressions arises as soon as one of the component events becomes active. To be more precise, we say that at a certain time $t$ the disjunction is active if at least one of the component events is active. If only one of the component events is active, its activation time-stamp becomes the activation time-stamp of the disjunction; if both the components events are active, the highest activation time-stamp of them is assumed to be the activation time-stamp of the disjunction.

For instance, as an example let us consider the sample event expression create(stock), modify (stock.quantity), two occurrences of the primitive event create (stock) at times $t_{1}$ and $t_{3}$, and one occurrence of the primitive event modify (stock.quantity) at time $t_{2}$, with $t_{1}<t_{2}<t_{3}$. At time $t<t_{1}$ the disjunction event is not active; at time $t_{1} \leq t<t_{2}$ the disjunction is active and its activation time-stamp is $t_{1}$; at time $t_{2}<t<t_{3}$, the disjunction is active and its activation time-stamp is now $t_{2}$; finally, at time $t \geq t_{3}$ the disjunction is active and its activation time-stamp is now $t_{3}$.

When we consider the conjunction $\mathcal{E}_{1}+\mathcal{E}_{2}$ of two events, it is intuitive that it is active when both of the component events are active. If so, the activation time-stamp is the highest of the activation time-stamps of the component events.

For instance, as an example let us consider the sample event expression create(stock) + modify (stock.quantity), two occurrences of the primitive event create (stock) at times $t_{1}$ and $t_{3}$, and one occurrence of the primitive event modify (stock. quantity) at time $t_{2}$, with $t_{1}<t_{2}<t_{3}$. At time $t<t_{1}$ the conjunction event is not active; at time $t_{1} \leq t<t_{2}$ the conjunction is still not active; at time $t_{2} \leq t<t_{3}$, the conjunction is active and its activation time-stamp is $t_{2}$; finally, at time $t \geq t_{3}$ the activation time-stamp is now $t_{3}$.

In complex applications it is often necessary to consider the absence of an event, i.e. one would like to check for the absence of occurrences of an event.

We think that a negation event $-\mathcal{E}$ is active when the negated event (also called component event) is not active; in particular, if there are no occurrences

\begin{tabular}{|l|c|c|}
\hline & Instance-Oriented & Set-Oriented \\
\hline Negation & $-=$ & - \\
\hline Conjunction & $+=$ & + \\
Precedence & $<=$ & $<$ \\
\hline Disjunction &,$=$ &, \\
\hline
\end{tabular}

Fig. 1. Composition Operators Set. 


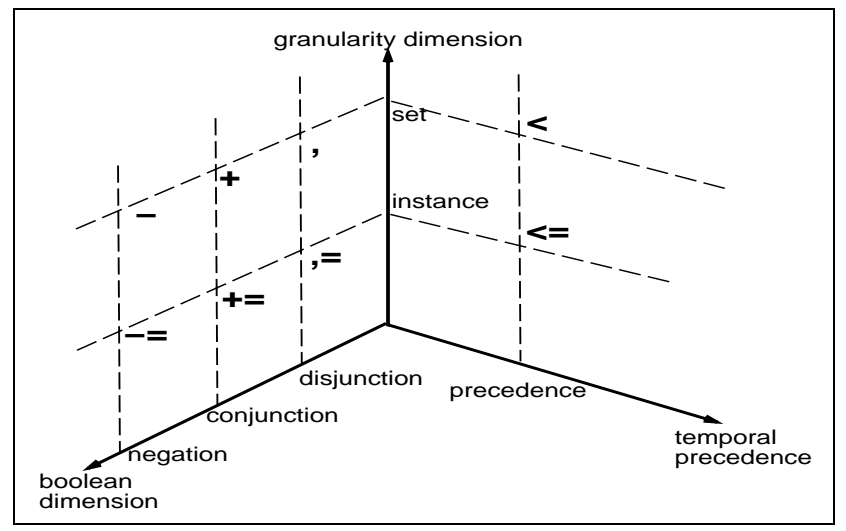

Fig. 2. Event operators dimensions

of the negated event at time $t$, the activation time-stamp is the current time.

For instance, let us consider the first occurrence of the event create(stock) at time $t_{1}$ and its negation, -create(stock). At time $t \geq t_{1}$, since the event create(stock) is active, the negation is not active; at time $t<t_{1}$, since create (stock) is not active, the negation is active and its activation time-stamp is $t$, because it is occurring at time $t$.

Similarly to the conjunction, the precedence $\mathcal{E}_{1}<\mathcal{E}_{2}$ of two event expressions is active provided that both the component events are active; moreover, the first component event must become active earlier than the second one.

For instance, as an example let us consider the sample event expression create (stock) < modify (stock.quantity), two occurrences of the primitive event create(stock) at times $t_{1}$ and $t_{3}$, and one occurrence of the primitive event modify (stock.quantity) at time $t_{2}$, with $t_{1}<t_{2}<t_{3}$. At time $t<t_{1}$ the precedence event is not active; at time $t_{1} \leq t<t_{2}$ the precedence is still not active; at time $t_{2} \leq t<t_{3}$, the precedence is active and its activation time-stamp is $t_{2}$; finally, at time $t \geq t_{3}$ the precedence is active and its activation time-stamp still remains at $t_{2}$, because the second creation has time-stamp greater than that of the last modification.

We are able to write any complex set-oriented event expression, e.g.

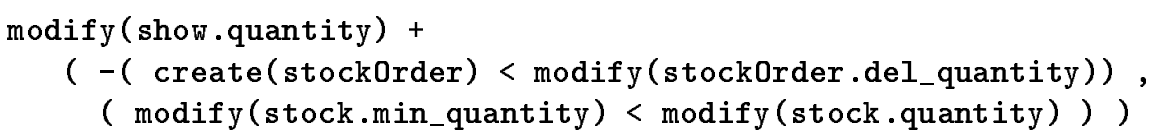

which is active if there is a modification of the product quantity on a shelf, and there is not a creation of a stock order followed by a modification of the delivered quantity for a stock order, or there is a modification of the minimum quantity for a stock followed by a modification of the quantity for a stock. 


\subsection{Instance-Oriented Operators}

Instance-Oriented operators are useful to catch the occurrence of composite events on the same object. For this reason, instance-oriented operators have higher priority than set-oriented ones, and they cannot be applied to event subexpressions obtained by means of set-oriented operators.

In contrast, an event expression obtained using instance-oriented operators can appear as an operand of a set-oriented operator; in fact, it is very intuitive to pass from the instance-oriented to the set-oriented level, as we will show later.

A primitive event occurs on an object $O$ when a new occurrence of that event type arises and affects $O$. As in the set-oriented case, at time $t$ the following situations are possible: no event occurrences of that type have arisen yet on $O$, so the primitive event is not active for $O$; at least one occurrence of that type has arisen on $O$, then the primitive event is active for $O$ and the activation time-stamp is that of the more recent occurrence. For instance, let us imagine that two occurrences of the event create (stock) arise at time $t_{1}$ and $t_{2}$ on the objects $O_{1}$ and $O_{2}$ respectively. At time $t<t_{1}$ the event is not active for both the objects; at time $t_{1} \leq t<t_{2}$ the event is active only for $O_{1}$ and its activation time-stamp is $t_{1}$; finally, at time $t_{2} \leq t$ the event is still active for $O_{1}$ with activation time-stamp $t_{1}$, but it becomes active for $O_{2}$ too and its activation time-stamp is $t_{2}$.

The instance-oriented conjunction $\mathcal{E}_{1}+=\mathcal{E}_{2}$ of two events on the same object $O$, is active when both the component events are active for $O$. The activation time-stamp for $O$ is the highest of the activation time-stamps for the component events. For instance, create(stock) $+=$ modify (stock.quantity) is an instance-oriented conjunction that becomes active for a stock object $O$ when $O$ has been created and its quantity has been changed. When used in a set-oriented expression, an instance-oriented conjunction is active if there is at least one object affected by the two component event expressions. For instance, consider the expression

modify (show.quantity) $+($ create(stock) $+=$ modify (stock.quantity))

which is active when a change of a shown product quantity occurs and at least a stock object has been created and its quantity modified.

The instance-oriented disjunction $\mathcal{E}_{1},=\mathcal{E}_{2}$ of two event expressions on an object $O$ intuitively arises as soon as one of the component events becomes active for $O$. Precisely, at a certain time $t$ the disjunction is active for $O$ if at least one of the component events is active for $O$. If only one of the component events is active for $O$, its activation time-stamp becomes the activation time-stamp of the disjunction; if both the components events are active for $O$, the highest activation time-stamp of them is assumed to be the activation time-stamp of the disjunction. Consider the expression create(stock), = modify (stock.quantity) as an example of instance-oriented disjunction, two occurrences of the primitive event create (stock) at times $t_{1}$ and $t_{3}$ on objects $O_{1}$ and $O_{3}$ respectively, and two occurrences of the event modify (stock .quantity) at time $t_{2}$ on objects $O_{1}$ and $O_{2}$ respectively, with $t_{1}<t_{2}<t_{3}$. At time $t<t_{1}$ the disjunction event is 
not active for all the three mentioned objects; at time $t_{1} \leq t<t_{2}$ the disjunction is active for $O_{1}$ with activation time-stamp $t_{1}$ and still not active for $O_{2}$ and $O_{3}$; at time $t_{2} \leq t<t_{3}$, the disjunction is still active for $O_{1}$ with activation time-stamp $t_{1}$ but is now active for $O_{2}$ with activation time-stamp $t_{2}$; finally, at time $t \geq t_{3}$ the disjunction is now active also for $O_{3}$ with activation time-stamp $t_{3}$. When used in a set-oriented expression, an instance-oriented disjunction is active if there is at least one object affected by the disjunction of the component event expressions. For instance, consider the expressions:

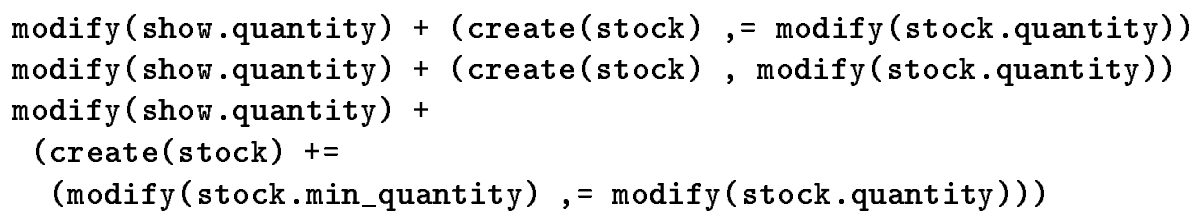

The first one is active when a change of a shown product quantity occurs and a stock object has been created or its quantity modified. Observe that with such a use the effect is the same as the second expression, that is active when a change of a shown product quantity occurs and there are a creation of a stock object or a modification of the quantity for a stock object, the two objects being possibly different; in fact, the instance-oriented disjunction operator has been introduced to be used in instance-oriented event expressions, based on a operator set that we want to be orthogonal w.r.t. set-oriented operator set. The third expression clarifies this concept, because it is active when a change of a shown product quantity occurs and there is a creation of a stock objecy on which either a modification of the minimum quantity or a modification of the quantity occur.

The instance-oriented negation $-=\mathcal{E}$ expresses the absence of occurrences of an event type for an object $O$ : it is active when the negated event is not active for $O$ and the activation time-stamp is the current time. For instance, let us consider two occurrences of the event create (stock) at time $t_{1}$ and $t_{2}$ affecting $O_{1}$ and $\mathrm{O}_{2}$ respectively, and the negation event -=create (stock). At time $t<t_{1}$ the negation is active for both $O_{1}$ and $O_{2}$, with activation time-stamp $t$ for both; at time $t_{1} \leq t<t_{2}$, since the event create (stock) is active for $O_{1}$ but not for $\mathrm{O}_{2}$, the negation is not active for $O_{1}$ but is still active for $O_{2}$ with activation time-stamp $t$; finally, at time $t_{2} \leq t$, since create (stock) is active for both, the negation is not active for both $\bar{O}_{1}$ and $O_{2}$. It is easy to think of the use of an instance-oriented negation in the following intuitive way: it is active if there is no object which the instance-oriented negation is active for, otherwise it is not active. Notice that if the $-=$ operator is applied to elementary event types, using it in a set-oriented expression leads to the same result as the set-oriented version; things change when it is applied to more complex instance-oriented expressions. For instance, consider the expressions:

modify (show.quantity) $+-=($ create (stock)+=modify (stock.quantity)) modify (show.quantity) $+-($ create (stock) + modify (stock.quantity))

The first one is active when a change of a shown product quantity occurs and no stock object has been created and its quantity modified. Instead, the second one is active when a change of a shown product quantity occurs and there is neither 
a creation of a stock object nor a modification of the quantity for a stock object, the two objects being possibly different.

Similarly to the conjunction, the instance-oriented precedence $\mathcal{E}_{1}<=\mathcal{E}_{2}$ of two events is active when both the component events are active on the same object $O$, the first one becoming active earlier than the second one. For instance, let us consider modify ( stock.min_quantity) <= modify ( stock.quantity), two occurrences of the event modify (stock.min_quantity) at times $t_{1}$ and $t_{3}$ on the same object $O_{1}$, and one occurrence of the event modify (stock.quantity) at time $t_{2}$ again on the object $O_{1}$, with $t_{1}<t_{2}<t_{3}$. At time $t<t_{1}$ the precedence event for $O_{1}$ is not active; at time $t_{1} \leq t<t_{2}$ the precedence is still not active for $O_{1}$; at time $t_{2} \leq t<t_{3}$, the precedence is active for $O_{1}$ and its activation time-stamp is $t_{2}$; finally, at time $t \geq t_{3}$ the precedence is active for $O_{1}$ and its activation time-stamp is still $t_{2}$. When used in a set-oriented expression, an instance-oriented precedence is active if there is at least one object affected by the sequence of the two component event expressions. For instance, consider the expressions:

modify (show.quantity) $+($ create(stock) $<=$ modify (stock.quantity)) modify (show.quantity) + (create (stock) < modify (stock.quantity))

The first one is active when a change of a shown product quantity occurs and at least a stock object has been created and later its quantity modified. Instead, the second one is active when a change of a shown product quantity occurs and there is a creation of a stock object followed by a modification of the quantity for a stock object, the two objects being possibly different.

\subsection{Event Formulas}

As introduced in Sections 3, event formulas (see Section 2) are extended in consequence of the introduction of the event language.

Event expressions. The occurred predicate now supports event expressions limited to instance-oriented operators. This is due to the semantics of the predicate: it returns all the objects affected by the specified event expression ${ }^{4}$. For example:

occurred (create(stock) <= modify(stock.quantity), X)

binds all the objects created whose attribute quantity has been modified to variable $\mathrm{X}$. Depending on the consumption mode selected for the rule, the above formula retrieves either all the objects affected by that particular combination

\footnotetext{
${ }^{4}$ Chimera supports also a predicate holds which composes event types. However, there is no need of such predicate in the new Chimera extended with event calculus, since event composition can be explicitily evaluated by the calculus. For instance, net effect for the creation operation in presence of sequences of modifications and deletions is given by the following event formula:

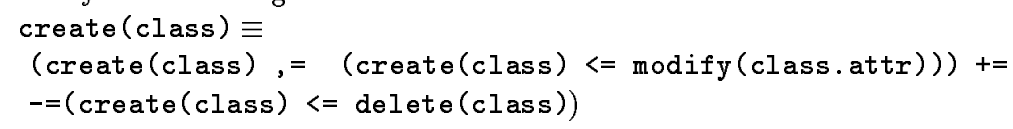


of event types since the beginning of the transaction (preserving rule) or only those affected since the last consideration of the rule (consuming rule). Observe that this is exactly the same semantics of Chimera without composite events, reviewed in Section 2.

Occurrence time-stamp. This new predicate is similar to the occurred predicate but it provides the time-stamp of the specified composite event occurrences as well. For example:

at $(\operatorname{create}($ stock) $<=\operatorname{modify}($ stock.quantity), $\mathrm{X}, \mathrm{T}$ )

where $T$ is a variable defined on type time.

Its semantics is defined as follows: given an object $\mathrm{X}, \mathrm{T}$ assumes all the timestamps, in the observed time interval, at which an occurrence of the specified event expression arises for that object. In the above example, if the creation of a stock object is followed by two updates of its quantity attribute, the specified composite event occurs twice, exactly when the two updates occur.

The observed time interval depends on the consumption mode selected for the rule: it can range either from the beginning of the transaction to the current time (preserving rules), or from the last consideration of the rule to the current time (consuming rule).

\section{Formal Semantics}

This Section is organized as follows: at first, we describe our approach to the definition of event calculus; then we precisely define our model of Event Base, on which the definitions presented later are based; then, we give the formal semantics for both set-oriented and instance-oriented operators; finally, triggering semantics is formulated in formal way.

Composite event semantics The main goal of our work is to provide the event language with a semantics that preserves boolean properties, such as De Morgan rules, when time properties associated to event occurrences (their time-stamp) are considered. In fact, event occurrences exist because they are generated at a certain time instant, thus that aspect should be always taken into account when event expressions are evaluated.

Event expressions are used in the event part of the rule and possibly in event formulas in the condition part. So the event occurrence determine whether the rule is triggered or not.

The main idea is the following: when a portion of the event base EB (the $\log$ of all events occurred since the beginning of the transaction, see Section 4.1) is investigated, for each primitive event type we construct a function dependent on time $t$, called the time-stamp of the more recent event occurrence in the investigated portion of $\mathrm{EB}$, indicated with $t s$. The $t s$ function of an event type is constructed on the basis of the positive time-stamp of the last occurrence of the event type, if an event occurrence exists in the investigated portion of EB. Otherwise, $t s$ calculated in $t$ is set to a negative value, equal to $-t$. 


\begin{tabular}{|c|l|c|c|}
\hline EID & event-type & OID & time-stamp \\
\hline$e_{1}$ & $\langle$ create, stock $\rangle$ & $o_{1}$ & $t_{1}$ \\
\hline$e_{2}$ & $\langle$ create, stock $\rangle$ & $o_{2}$ & $t_{2}$ \\
\hline$e_{3}$ & $\langle$ create, order $\rangle$ & $o_{3}$ & $t_{3}$ \\
\hline$e_{4}$ & $\langle$ create, notFilledOrder $\rangle$ & $o_{3}$ & $t_{3}$ \\
\hline$e_{5}$ & $\langle$ modify, stock, quantity $\rangle$ & $o_{1}$ & $t_{4}$ \\
\hline$e_{6}$ & $\langle$ modify, stock, quantity $\rangle$ & $o_{2}$ & $t_{4}$ \\
\hline$e_{7}$ & $\langle$ delete, stock $\rangle$ & $o_{1}$ & $t_{5}$ \\
\hline
\end{tabular}

Fig. 3. Example of EB.

Thus, the sign of the $t s$ function of an event type states whether an occurrence of that event type exists in the portion of EB relevant for rule triggering: if positive, an event type occurrence exists; if negative, otherwise. Consequently, it is sufficient to determine an instant $t$ in which function $t s$ is positive to solve rule triggering.

When dealing with negation, the intuition is that $t s$ function of a negation event calculated at time $t$ has the opposite value of $t s$ of the negated component event, for each time value. In fact if an occurrence of an event type does not exist in the portion of $\mathrm{EB}$ relevant for rule triggering (i.e. all event occurrences more recent than the last consideration of the rule), from the instant of the last consideration of the rule, $t s$ value of the negated event at time $t$, is the time value $t$. It comes that $t s$ functions of primitive event types are calculated by a simple lookup into a portion of EB.

From these basic $t s$ functions, our event calculus algebraically derives $t s$ functions for event expressions from $t s$ functions associated to its primitive components. As already said, these expressions are obtained applaying arbitrarily boolean operators and precedence operator to primitive event types. Derived $t s$ functions associated to event expressions have the same properties of $t s$ functions for primitive event types.

\subsection{The Event Base}

The Event Base (EB) is the log containing all the event occurrences since the beginning of the transaction. In this paper we model the EB as a table having the structure depicted in Figure 3.

Each row contains an event occurrence, characterized by its unique identifier (EID), the event type, the Object Identifier (OID) of the object affected by the event occurrence, and the time-stamp of the time instant the event occurred at. The event type is described by the name of the command that changed the object state, possibly followed by the object class name and an attribute name. In the following, we refer to the EID of a generic event occurrence as $e$.

Given an event occurrence $e$, we can define a set of useful functions returning properties of $e$ stored in the EB. Figure 4 contains examples of the defined functions; derived from the EB state of Figure 3. 


\begin{tabular}{||l|l||}
\hline type $: e_{1} \rightarrow\langle$ create, stock $>$ & obj $: e_{4} \rightarrow o_{3}$ \\
type $: e_{5} \rightarrow\langle$ modify, stock, quantity $>$ & obj: $e_{5} \rightarrow o_{1}$ \\
type $: e_{6} \rightarrow<$ delete, stock $>$ & obj: $e_{6} \rightarrow o_{2}$ \\
\hline timestamp: $e_{2} \rightarrow t_{2}$ & eventonclass $: e_{1} \rightarrow$ stock \\
timestamp $: e_{3} \rightarrow t_{3}$ & eventonclass $: e_{5} \rightarrow$ stock \\
timestamp $: e_{4} \rightarrow t_{3}$ & \\
\hline
\end{tabular}

Fig. 4. Examples of event attribute matches on events in EB.

type type : EID $\rightarrow$ eventtype

This function matches each event occurence to its event type.

obj obj : $E I D \rightarrow O I D$

This relation matches each event occurrence to the object whose state has been modified by that event.

timestamp timestamp: EID $\rightarrow$ time

This function matches each event occurrence to its time-stamp.

eventonclass eventonclass $: E I D \rightarrow$ classname

This function matches each event occurrence to the class to which the object affected by the event occurrence belongs. Note that this piece of information is part of the event-type attribute.

\subsection{Set-Oriented Case}

The definition of $t s$ of a primitive event type $\mathcal{E}$ at time $t$ is:

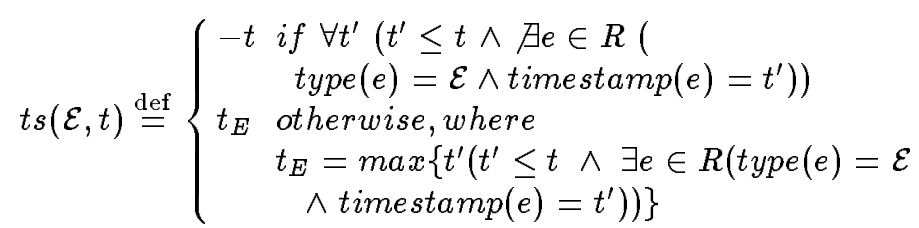

where $R$ is the set of event occurrences to which the event calculus applies.

We also introduce the function $\mathrm{u}(\mathrm{t}): u(t) \stackrel{\text { def }}{=} 0$ if $t \geq 0, u(t) \stackrel{\text { def }}{=} 1$ if $t \geq 0$.

From the above definitions, the presence of an event occurrence in $R$ at time $t$, is expressed by the logical predicate $\operatorname{occ}(\mathcal{E}, t)$ which is true if $u(t s(\mathcal{E}, t))=1$, false otherwise.

As already said informally, the semantics of negation is $t s(-\mathcal{E}, t) \stackrel{\text { def }}{=}-t s(\mathcal{E}, t)$.

Semantics of the other set-oriented operators is given in two steps: at first, we give a precise definition in logical style; second, that definition is translated into an algebraic equivalent expression that can be used for the evaluation of the $t s$ function associated to the overall event expression. 


\section{LogicalStyleSemantics}

\begin{tabular}{|c|c|}
\hline 1) $t s(\mathcal{A}+\mathcal{B}, t) \stackrel{\text { def }}{=}$ & $\begin{array}{l}\min \{t s(\mathcal{A}, t), t s(\mathcal{B}, t)\} \text { if } \neg \text { occ }(\mathcal{A}, t) \vee \neg o c c(\mathcal{B}, t) \\
\max \{t s(\mathcal{A}, t), t s(\mathcal{B}, t)\} \text { if } \operatorname{occ}(\mathcal{A}, t) \wedge \operatorname{occ}(\mathcal{B}, t)\end{array}$ \\
\hline 2) $t s((\mathcal{A}, \mathcal{B}), t) \stackrel{\text { def }}{=}$ & $\left\{\begin{array}{l}\min \{t s(\mathcal{A}, t), t s(\mathcal{B}, t)\} \text { if } \neg \text { occ }(\mathcal{A}, t) \wedge \neg o c c(\neg \mathcal{B}, t) \\
\max \{\operatorname{ts}(\mathcal{A}, t), t s(\mathcal{B}, t)\} \text { if occ }(\mathcal{A}, t) \vee \operatorname{occ}(\mathcal{B}, t)\end{array}\right.$ \\
\hline 3) $t s((\mathcal{A}<\mathcal{B}), t) \stackrel{\text { def }}{=}$ & 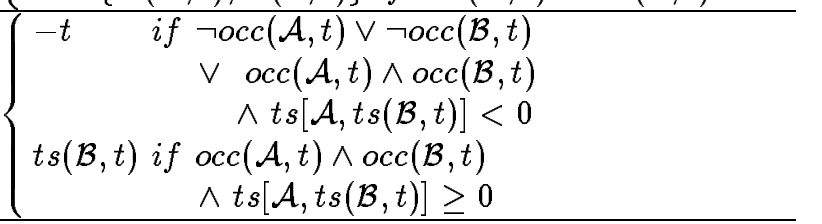 \\
\hline \multicolumn{2}{|c|}{$\begin{array}{c}\text { AlgebraicSemantics } \\
\end{array}$} \\
\hline \multicolumn{2}{|c|}{ 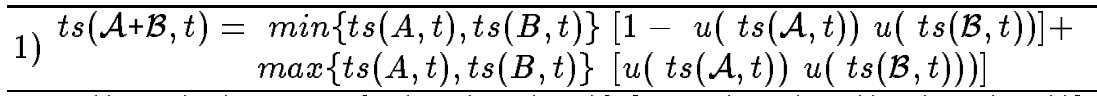 } \\
\hline \multicolumn{2}{|c|}{$\begin{aligned} 2_{2} t s((\mathcal{A}, \mathcal{B}), t)= & \max \{t s(\mathcal{A}, t), t s(\mathcal{B}, t)\}[1-u(-t s(\mathcal{A}, t)) u(-t s(\mathcal{B}, t))]+ \\
& \min \{t s(\mathcal{A}, t), t s(\mathcal{B}, t)\}[u(-t s(\mathcal{A}, t)) u(-t s(\mathcal{B}, t))]\end{aligned}$} \\
\hline & $\begin{array}{l}-t[1-u(\operatorname{ts}(\mathcal{B}, t)) u(t s(\mathcal{A}, t s(\mathcal{B}, t)))]+ \\
s(\mathcal{B}, t)[u(t s(\mathcal{B}, t)) u(t s(\mathcal{A}, t s(\mathcal{B}, t)))]\end{array}$ \\
\hline
\end{tabular}

It is possible to show that several properties holds, like the De Morgan property that $t s(-((-\mathcal{A})+(-\mathcal{B})), t)$ is equivalent to $t s((\mathcal{A}, \mathcal{B}), t)$.

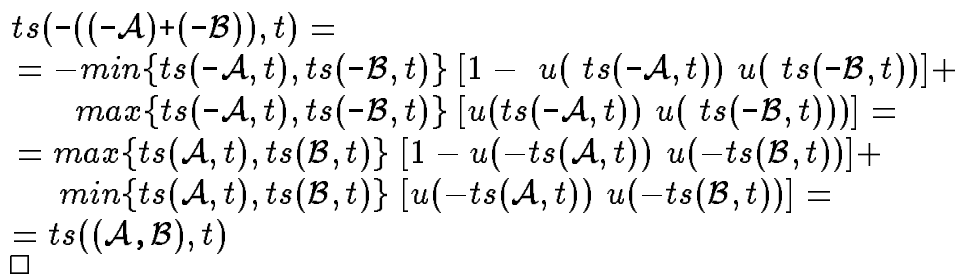

A graphical proof of this property is shown in Figure 5: for a set of event occurrences (of type $A, B$, and $C$, where event type $C$ is not involved in the expression), it shows $t s$ functions for both primitive and complex event expressions used in De Morgan proof.

The dual De Morgan property $t s((\mathcal{A}+\mathcal{B}), t)=t s(-(-\mathcal{A},-\mathcal{B}), t)$ and the following properties can be proved analogously.

$$
\begin{array}{l|l}
\mathcal{E}_{1}+\mathcal{E}_{2}=\mathcal{E}_{2}+\mathcal{E}_{1} & \left(\mathcal{E}_{1}+\mathcal{E}_{2}\right)<\mathcal{E}_{3}=\left(\mathcal{E}_{1}<\mathcal{E}_{3}\right)+\left(\mathcal{E}_{2}<\mathcal{E}_{3}\right) \\
\mathcal{E}_{1}, \mathcal{E}_{2}=\mathcal{E}_{2}, \mathcal{E}_{1} & \left(\mathcal{E}_{1}, \mathcal{E}_{2}\right)<\mathcal{E}_{3}=\left(\mathcal{E}_{1}<\mathcal{E}_{3}\right),\left(\mathcal{E}_{2}<\mathcal{E}_{3}\right) \\
\hline\left(\mathcal{E}_{1}+\mathcal{E}_{2}\right)+\mathcal{E}_{3}=\mathcal{E}_{1}+\left(\mathcal{E}_{2}+\mathcal{E}_{3}\right) & \mathcal{E}_{1}<\left(\mathcal{E}_{2}+\mathcal{E}_{3}\right)=\left(\mathcal{E}_{1}<\mathcal{E}_{2}\right),\left(\mathcal{E}_{1}<\mathcal{E}_{3}\right) \\
\left(\mathcal{E}_{1}, \mathcal{E}_{2}\right), \mathcal{E}_{3}=\mathcal{E}_{1},\left(\mathcal{E}_{2}, \mathcal{E}_{3}\right) & \mathcal{E}_{1}<\left(\mathcal{E}_{2}, \mathcal{E}_{3}\right)=\left(\mathcal{E}_{1}<\mathcal{E}_{2}\right)+\left(\mathcal{E}_{1}<\mathcal{E}_{3}\right) \\
\left.\hline \mathcal{E}_{1}+\left(\mathcal{E}_{2}, \mathcal{E}_{3}\right)\right)=\mathcal{E}_{1}+\mathcal{E}_{2}, \mathcal{E}_{1}+\mathcal{E}_{3} & \mathcal{E}_{1}<\mathcal{E}_{2}<\mathcal{E}_{3}=\left(\mathcal{E}_{1}<\mathcal{E}_{2}\right)<\mathcal{E}_{3}
\end{array}
$$

\subsection{Instance-Oriented Case}

Instance-Oriented operators are useful to catch the occurrence of composite events on the same object. For this reason, instance-oriented operators have higher priority than set-oriented ones, and they cannot be applied to event subexpressions obtained by means of set-oriented operators. 


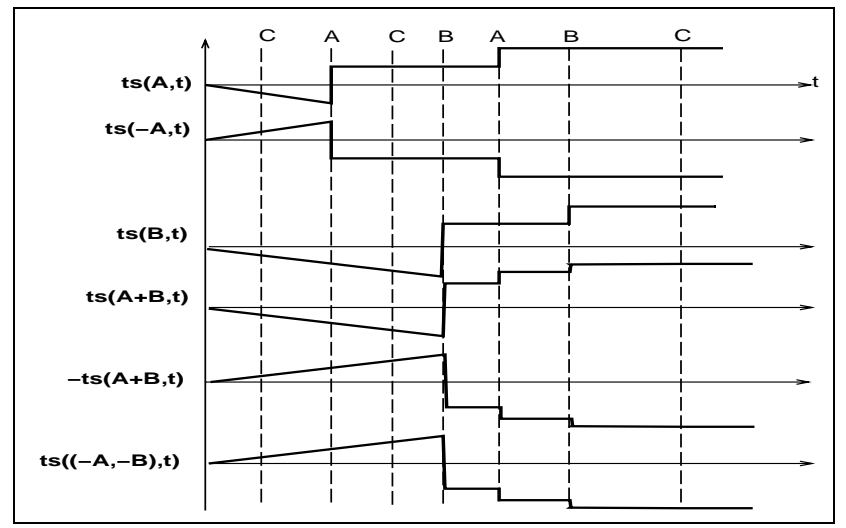

Fig. 5. Examples of $t s$ functions with event expression

The exposition will follow a schema similar to that of Section 4.2: after the introduction of basic definitions and instance-oriented composition operators, we show how instance-oriented event expressions are evaluated inside set-oriented expressions.

In the instance-oriented case we make use of ots functions, which are very similar to $t s$ functions, except for the fact that they refer to a single object.

$$
\operatorname{ots}(\mathcal{E}, t, o i d) \stackrel{\text { def }}{=}\left\{\begin{array}{rr}
-t \quad \text { if } \forall t^{\prime}\left(t^{\prime} \leq t \wedge \nexists e \in R(\operatorname{type}(e)=\mathcal{E}\right. \\
\left.\left.\quad \wedge t \text { imestamp }(e)=t^{\prime} \wedge \text { obj }(e)=\text { oid }\right)\right) \\
t_{E} \quad \begin{array}{r}
\text { otherwise, where } \\
t_{E}=\max \left\{t ^ { \prime } \left(t^{\prime} \leq t \wedge \exists e \in R(\text { type }(e)=\mathcal{E}\right.\right. \\
\left.\left.\left.\wedge \text { timestamp }(e)=t^{\prime} \wedge \text { obj }(e)=\text { oid }\right)\right)\right\}
\end{array}
\end{array}\right.
$$

where $R$ is the set of event occurrences to which the event calculus applies. As in the set-oriented case, it is $\operatorname{oocc}(\mathcal{E}, t$, oid $)=$ true if $u($ ots $(\mathcal{E}, t)$, oid $)=1$, false otherwise.

\begin{tabular}{c} 
Conjunction : LogicalStyleSemantics \\
\hline ots $(\mathcal{A}+=\mathcal{B}, t$, oid $) \stackrel{\text { def }}{=}$ \\
$\left\{\begin{array}{l}\min \{\text { ots }(\mathcal{A}, t, \text { oid }), \text { ots }(\mathcal{B}, t, \text { oid })\} \text { if } \neg \operatorname{oocc}(\mathcal{A}, t, \text { oid }) \vee \neg \operatorname{oocc}(\mathcal{B}, t, \text { oid }) \\
\max \{\text { ots }(\mathcal{A}, t, \text { oid }), \text { ots }(\mathcal{B}, t, \text { oid })\} \text { if } \operatorname{oocc}(\mathcal{A}, t, \text { oid }) \wedge \text { oocc }(\mathcal{B}, t, \text { oid })\end{array}\right.$ \\
Conjunction : AlgebraicSemantics \\
ots $(\mathcal{A}+=\mathcal{B}, t$, oid $)=$ \\
$\min \{$ ots $(A, t$, oid $)$, ots $(B, t$, oid $)\}[1-u($ ots $(\mathcal{A}, t$, oid $)) u($ ots $(\mathcal{B}, t$, oid $))]+$ \\
$\max \{$ ots $(A, t$, oid $)$, ots $(B, t$, oid $)\}[u($ ots $(\mathcal{A}, t$, oid $)) u($ ots $(\mathcal{B}, t$, oid $)))]$
\end{tabular}

The disjunction, negation and precedence operators are similarily extended to the instance-oriented case, and expressed respectively with ",=", "-=" and "<=". So all the properties valid for the set-oriented operators, can be easily extended to the instance-oriented case. 
We now show how ots functions are related to $t s$ functions to be evaluated inside set-oriented expressions, and which properties can be proved.

\begin{tabular}{|c|l|}
\hline \multirow{5}{*}{ ots to ts } & $t s(\mathcal{A}+=\mathcal{B}, t)=\min \{$ ots $(\mathcal{A}+=\mathcal{B}, t$, oid $)\}, \forall$ oid $\in R$ \\
& $t s(\mathcal{A}<=\mathcal{B}, t)=\min \{$ ots $(\mathcal{A}<=\mathcal{B}, t$, oid $)\}, \forall$ oid $\in R$ \\
& $t s(\mathcal{A},=\mathcal{B}, t)=\min \{$ ots $(\mathcal{A}+=\mathcal{B}, t$, oid $)\}, \forall$ oid $\in R$ \\
& $t s(-=\mathcal{A}, t)=\max \{$ ots $(-=\mathcal{A}, t$, oid $)\}, \forall$ oid $\in R$ \\
\hline \multirow{3}{*}{ properties } & ots $(\mathcal{A}, t$, oid $) \leq t s(\mathcal{A}, t) \forall$ oid \\
& $t s(\mathcal{A}+=\mathcal{B}, t) \leq t s(\mathcal{A}+\mathcal{B}, t) \quad t s(\mathcal{A},=\mathcal{B}, t) \leq t s(\mathcal{A}, \mathcal{B}, t)$ \\
& $t s(\mathcal{A}<=\mathcal{B}, t) \leq t s(\mathcal{A}<\mathcal{B}, t) \quad t s(-=\mathcal{A}, t) \geq t s(-\mathcal{A}, t)$ \\
\hline
\end{tabular}

\subsection{Specification of rule triggering}

The formal specification of rule triggering at time $t$ for a rule $r$ is given by the predicate $T(r, t)$ : if the result of its evaluation is true, then the rule is triggered.

$$
\begin{aligned}
T(r, t) \stackrel{\text { def }}{=} & R=\left\{e \mid e \in E B \wedge r . t_{0}<t \text { timestamp }(e) \leq t\right\} \wedge \\
& R \neq \emptyset \wedge \exists t^{\prime}\left(r . t_{0}<t^{\prime} \leq t \wedge t s\left(r . \mathcal{E}, t^{\prime}\right)>0\right)
\end{aligned}
$$

where $r . t_{0}$ is the time-stamp of the last consideration of the rule, while $r . \mathcal{E}$ is the triggering event expression of the rule. Observe that the predicate defines the set $R$ which the $t s$ function must be applied to as the set of all event occurrences more recent than the last consideration of the rule: in fact, the event calculus can be applied to a generic set of event occurrences; orthogonally, the triggering semantics defines this set.

Note that intuitively this semantics implies that a rule can be triggered only if something happened, otherwise the triggering mechanism ends because there is nothing which rules can react to. The reason of this choice $(R \neq \emptyset)$ is that removing this constraint, a rule triggered by negated event types would always be fired even in absence of new event occurrences; then the system would become active instead of being reactive.

\section{Implementation}

The introduction of the event calculus language does not change the general architecture of the implementation of Chimera, described in [3], but affects only some specialized component, like the Event Handler and the Trigger Support: the former deals with event occurrences and stores them into the Occurred Events data structure; the latter maintains the current status of active rules (called triggers and chooses the trigger to be executed among those activated.

Chimera has a component, called Block Executor, which executes non interruptable execution blocks (user transaction lines or rule actions), finishes the execution of a block, it sends all the last generated event occurrences to the Event Handler in order to store them into the Occurred Events data structure. This data structure is maintaned as an event tree whose leaves are lists of event occurrences of the same type; furthermore, each leaves keeps the time-stamp of the more recent occurrence of the associated event type. 
At this moment, the Event Handler calls the Trigger Support whose task is the determination of new activated rules. The Trigger Support maintains in the Rule Table the current status of all defined rules; this table is managed by means of a hash table, for fast access, but rules are also linked together by means of a queue on the basis of the priority order. To deal with composite events, each rule has two time-stamps associated to it: one, called last-consideration, stores the last consideration time-stamp; the other, called last-consumption, stores the time-stamp of the last event consumption, which is either the last consideration time if the rule is consuming or the initial time-stamp of the transaction if the rule is preserving. Another flag associated to a rule is the triggered flag, set to true if the rule is triggered or to false otherwise.

The Trigger Support checks for activated rules in the following way. It looks up into the Rule Table for all rules which are not triggered. When it finds one, it computes the $t s$ value for the associated triggering event expression: if the computed value is positive, the rule is then triggered and the triggered flag is set to true (the rule will be detriggered once after its consideration).

Once new triggered rules are determined, the one to be executed is chosen by means of the rule queue, and passed to the Block Executor.

The evaluation of $t s$ should take into account a certain number of things. At first, to determine the $t s$ of a primitive event type is sufficient to query the occurred events table to get the last occurrence time-stamp of the desired event type $E$ : if this time-stamp is not less than the value of last-consideration, this is the value of $t s(E, t)$, otherwise $t s(E, t)$ value is $-t$ (where $t$ is the current timestamp). Second, when dealing with instance-oriented operators, it is necessary to keep trace of all monitored events occurred on a single object: to do that, a sparse data structure can be associated to each rule and maintained until the consideration, then it is made empty; each item in this data structure stores the OID of an object affected by some event type since the last consideration and the list of event occurrences affecting that object since the last consideration.

\subsection{Static Optimization}

In general, the computation of the $t s$ function for a given rule is an expensive task, especially if a large rule set has been defined. Our approach is to reduce the $t s$ recomputation, by doing it only when it is highly probable that $t s$ value becomes positive. The goal of the static optimization is to extract conditions on an event expression that guarantees, if not met, that the value of $t s$ cannot become positive (recall rule triggering condition). This analysis should be performed when a rule is defined, and its results used to drive the Trigger Support in determining triggered rules.

The occurrence of composite event type $\mathcal{E}$, at time $t$, is indicated by the fact that the associated function $t s$ assumes a new positive value at time $t$; thus, we need to check positive variations of $t s$, that we indicate as $\Delta^{+}(\mathcal{E})$. Depending on the composition operator, it may depend on positive or negative (indicated with $\Delta^{-}(\mathcal{E})$ ) variations of the component event expressions: the first case arises with conjunction, disjunction and precedence, the second one with negation. 


\begin{tabular}{|c|c|}
\hline $\begin{array}{l}\Delta^{+}(-\mathcal{E}) \rightarrow \Delta^{-}(\mathcal{E}) \\
\Delta^{-}(-\mathcal{E}) \rightarrow \Delta^{+}(\mathcal{E}) \\
\Delta^{+}\left(\mathcal{E}_{1}<\mathcal{E}_{2}\right) \rightarrow \Delta^{+}\left(\mathcal{E}_{2}\right) \\
\Delta^{-}\left(\mathcal{E}_{1}-\mathcal{E}_{2}\right) \rightarrow \Delta^{-}\left(\mathcal{E}_{2}\right) \\
\Delta^{+}\left(\mathcal{E}_{1} \text { bin-op } \mathcal{E}_{2}\right) \rightarrow \Delta^{+}\left(\mathcal{E}_{1}\right), \Delta^{+}\left(\mathcal{E}_{2}\right) \\
\Delta^{-}\left(\mathcal{E}_{1} \text { bin-op } \mathcal{E}_{2}\right) \rightarrow \Delta^{-}\left(\mathcal{E}_{1}\right), \Delta^{-}\left(\mathcal{E}_{2}\right)\end{array}$ & 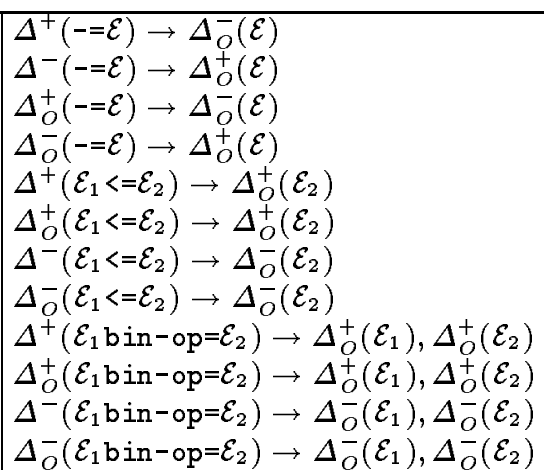 \\
\hline
\end{tabular}

Fig. 6. Derivation Rules.

\begin{tabular}{|l|l|}
$\left\{\Delta_{O}^{+}(\mathcal{E}), \Delta_{O}^{-}(\mathcal{E})\right\} \rightarrow\left\{\Delta_{O}(\mathcal{E})\right\}$ & $\left\{\Delta^{-}(\mathcal{E}), \Delta_{O}^{+}(\mathcal{E})\right\} \rightarrow\{\Delta(\mathcal{E})\}$ \\
$\left\{\Delta_{O}(\mathcal{E}), \Delta_{O}^{+}(\mathcal{E})\right\} \rightarrow\left\{\Delta_{O}(\mathcal{E})\right\}$ & $\left\{\Delta_{O}(\mathcal{E}), \Delta^{+}(\mathcal{E})\right\} \rightarrow\{\Delta(\mathcal{E})\}$ \\
$\left\{\Delta_{O}(\mathcal{E}), \Delta_{O}^{-}(\mathcal{E})\right\} \rightarrow\left\{\Delta_{O}(\mathcal{E})\right\}$ & $\left\{\Delta_{O}(\mathcal{E}), \Delta^{-}(\mathcal{E})\right\} \rightarrow\{\Delta(\mathcal{E})\}$ \\
$\left\{\Delta^{+}(\mathcal{E}), \Delta_{O}^{+}(\mathcal{E})\right\} \rightarrow\left\{\Delta^{+}(\mathcal{E})\right\}$ & $\left\{\Delta^{+}(\mathcal{E}), \Delta^{-}(\mathcal{E})\right\} \rightarrow\{\Delta(\mathcal{E})\}$ \\
$\left\{\Delta^{-}(\mathcal{E}), \Delta_{O}^{-}(\mathcal{E})\right\} \rightarrow\left\{\Delta^{-}(\mathcal{E})\right\}$ & $\left\{\Delta^{+}(\mathcal{E}), \Delta(\mathcal{E})\right\} \rightarrow\{\Delta(\mathcal{E})\}$ \\
$\left\{\Delta^{+}(\mathcal{E}), \Delta_{O}^{-}(\mathcal{E})\right\} \rightarrow\{\Delta(\mathcal{E})\}$ & $\left\{\Delta^{-}(\mathcal{E}), \Delta(\mathcal{E})\right\} \rightarrow\{\Delta(\mathcal{E})\}$ \\
\hline
\end{tabular}

Fig. 7. Simplification Rules.

This process can be performed until primitive event types are reached using a proper set of derivation rules (see Figure 6). In these rules for simplicity, we have used the symbol "bin-op" to indicate either the conjunction or the disjunction operator. These rules consider the instance-oriented operators as well; in order to deal with them, they use the symbols $\Delta_{O}^{+}(\mathcal{E}), \Delta_{O}^{-}(\mathcal{E})$ and $\Delta_{O}(\mathcal{E})$, which are analougous to the previous ones, but indicating ots variations for a single object. In the end, it leads to a set $V(\mathcal{E})$ of variations (positive or negative) for primitive event types describing whether or not the value of $t s$ must be recomputed, because it might have changed, when new event occurrences arise; in practice, the conditions described by $V(\mathcal{E})$ are sufficient conditions ensuring that if new arising event occurrences do not match $V(\mathcal{E})$, no recomputation of $t s$ is required.

Set $V(\mathcal{E})$ can be simplified using rules in Figure 7; in particular, with the symbol $\Delta(\mathcal{E})$ both a positive and negative variation is indicated. As an example, consider the following event expression $\mathcal{E}$.

$$
\mathcal{E} \equiv((A+B),(C+(-A))+((A+=C)<=(-=(B(<=A))
$$

The $V(\mathcal{E})$ set is obtained applying at first the derivation rules, then the simplification rules, as shown below. 


$$
\begin{aligned}
V(\mathcal{E}) & =\Delta^{+}(\mathcal{E})= \\
& =\left\{\Delta^{+}((A+B),(C+(-A))), \Delta^{+}((A+=C)<=(-=(B(<=A))\}=\right. \\
& =\left\{\Delta^{+}(A), \Delta^{+}(B), \Delta^{+}(C), \Delta^{-}(A), \Delta_{O}^{+}(A+=C), \Delta_{o}^{-}(B(<=A)\}=\right. \\
& =\left\{\Delta^{+}(A), \Delta^{+}(B), \Delta^{+}(C), \Delta^{-}(A), \Delta_{O}^{+}(A), \Delta_{o}^{+}(C), \Delta_{o}^{-}(B), \Delta_{o}^{-}(A)\right\}= \\
& =\left\{\Delta(A), \Delta(B), \Delta^{+}(C)\right\}
\end{aligned}
$$

\section{Conclusions}

This paper has proposed an extension of event calculus for Chimera, characterized by the following features:

- It requires a minimal set of orthogonal operators.

- It continuously evolves the semantics of Chimera by enabling more sophisticated rule triggering, while preserving the other semantic features of the rule system.

- It supports a formal and efficient evaluation of triggering caused by event expressions of arbitrary complexity, based on the use of a function ts which associates each event expression to an integer value; a rule is triggered when the corresponding ts expression is positive, and not triggered otherwise.

- The function $t s$ is assigned in such a way that certain obvious properties of calculus hold, such as De Morgan's rules or distributivity, associativity, and factoring of precedence expressions. Although this requirement seems mandatory to us, indeed it is not explicitly demontrated by some other event calculus proposals in the literature; achieving this result has required to us a nonobvious "twisting" of the ts functions.

- As an optimization, the evaluation of the $t s$ function is required when certain operations occur which have the potential of "changing the sign" of $t s$, and can be skipped otherwise.

Given the above features, we believe that the proposed event calculus applies not only to Chimera, but also to all other systems which currently support individual or disjunctive events (including all relational products which support triggers).

\section{References}

1. H. Branding, A. Buchmann, T. Kudrass, and J. Zimmermann. Rules in an open system: The reach rule system. In Proc. of the 1 st Int. Workshop on Rules in Database Systems, pages 127-142, Edimburgh, August 1993.

2. H. Branding, A. Buchmann, T. Kudrass, and J. Zimmermann. Rules in an open system: The reach rule system. In Proc. of the 1 st Int. Workshop on Rules in Database Systems, pages 111-125, Edimburgh, August 1993.

3. S. Castangia, G. Guerrini, D. Montesi, and G. Rodriguez. Design and implementation for the active rule language of chimera. In DEXA-95 6th international Workshop and Conference on Database and Expert Systems Applications, London, UK, September 1995. 
4. S. Ceri, P. Fraternali, S. Paraboschi, and L. Tanca. Active rule management in chimera. In [23].

5. S. Ceri and R. Manthey. Consolidated specification of chimera. Technical Report IDEA DE.2P.006.01, November 1993.

6. S. Chakravarthy, E. Anwar, L Maugis, and D. Mishra. Design of sentinel: an object-oriented dbms with event-based rules. Information and Software Technology, 36(9), 1994.

7. S. Chakravarthy, V. Krishnaprasad, E. Anwar, and S. K. Kim. Composite events for active databases: Semantics, context and detection. In Proceedings of the 20th International Conference on Very Large Data Bases, pages 606-617, Santiago, Chile, September 1994.

8. U. Dayal, A. P. Buchmann, and S. Chakravarthy. The hipac project. In [23].

9. U. Dayal, A. P. Buchmann, and D. R. McCarthy. Rules are objects too: A knowledge model for an active object-oriented database system. In K. R. Dittrich, editor, Proceedings of the 2nd International Workshop on Object-Oriented Databases. Springer-Verlag, 1988. LNCS 334.

10. P. Fraternali and L. Tanca. A structured approach for the definition of the semantics of active databases. June 1995. submitted to ACM-TODS.

11. S. Gatziu and K. R. Dittrich. Events in an active object-oriented database system. In Proc. of the 1st Int. Workshop on Rules in Database Systems, pages 23-39, Edimburgh, August 1993.

12. N. H. Gehani and H. V. Jagadish. Ode as an active database: Constraints and triggers. In Proceedings of the 17th International Conference on Very Large Data Bases, pages 327-336, Barcelona, Spain, September 1991.

13. N. H. Gehani, H. V. Jagadish, and O. Shmueli. Composite event specification in active databases: Model and implementation. In Proceedings of the 18th International Conference on Very Large Data Bases, pages 327-338, Vancouver, Canada, 1992. British Columbia.

14. N. H. Gehani, H. V. Jagadish, and O. Shmueli. Event specification in an active object-oriented database. In 1992 ACM SIGMOD, pages 81-90, San Diego, CA, USA, May 1992.

15. E. N. Hanson. Rule condition testing and action execution in ariel. In Proceedings of the 17th International Conference on Very Large Data Bases, pages 327-336, Barcelona, Spain, September 1991.

16. E. N. Hanson, M. Chaabouni, C-H. Kim, and Y-W. Wang. A predicate matching algorithm for database rule systems. ACM Journal, pages 271-280, May $199 \mathrm{O}$.

17. ISO-OSI. SQL3 Document X3H2-94-080 and SOU-003, ISO-ANSI Working Draft, 1994.

18. R. Maiocchi and B. Pernici. Temporal data management systems: A comparative view. IEEE Transactions on Knowledge and Data Engineerging, 3(4):504-524, December 1991.

19. W. Naqvi and M. T. Ibrahim. Rule and knowledge management in an active database system. In [23].

20. N. W. Paton, O. Diaz, M. H. Williams, J. Campin, A. Dinn, and A. Jaime. Dimensions of active behaviour. In Proc. of the 1 st Int. Workshop on Rules in Database Systems, pages 40-57, Edimburgh, August 1993.

21. M. Stonebraker, A. Jhingran, J. Goh, and S. Potamios. On rules, procedures, chaching, and views in data base systems. In Proc.ACM-SIGMOD Int. Conference, pages 281-290, Atlantic City, June 1990. 
22. M. Teisseire, P. Poncelet, and R. Cicchetti. Towards event-driven modelling for database design. In Proceedings of the 20th International Conference on Very Large Data Bases, pages 327-336, Santiago, Chile, September 1994.

23. J. Widom and S. Ceri. Active Database Systems. Morgan Kaufmann, San Matteo, California, August 1995.

24. J. Widom, R. J. Cohrane, and B. G. Lindsay. Implementing set-oriented production rules as an extension od starburst. In Proceedings of the 17th International Conference on Very Large Data Bases, pages 275-285, Barcelona, Spain, September 1991.

25. J. Widom and S. J. Finkelstein. Set-oriented production rules in relational database systems. In Proc.ACM-SIGMOD Int. Conference, pages 250-270, Atlantic City, June 1990. 